\title{
Which urinary diversion is best after radical cystectomy? The case for "incontinent" diversion
}

\author{
Michael J. Leveridge, MD, FRCSC; ${ }^{*}$ Michael A.S. Jewett, MD FRCSC ${ }^{\dagger}$
}

$\mathrm{T}$ he primary indication for continent urinary diversion after radical cystectomy is an improved quality of life (QOL). Proponents of expanded indications for continent diversion claim that patients enjoy an improved QOL without a stoma and a bulging, urine-filled collection bag. This would seem intuitive, but health related QOL data do not support this belief. ${ }^{1-4}$ Several studies have been undertaken to characterize QOL changes in patients after cystectomy and urinary diversion. Unfortunately, few offer prospective or baseline data, and most are single-centre series. The highly regarded and experienced group of Hart, Skinner and colleagues ${ }^{2}$ noted no differences in QOL between groups of patients with ileal conduits and cutaneous or urethral Kock pouches following radical cystectomy. The Mainz group noted good satisfaction, regardless of chosen diversion, in a prospective series of patients undergoing both diversions. ${ }^{3}$ A review from Vanderbilt University ${ }^{4}$ demonstrated a modest QOL improvement in patients with orthotopic neobladders, but this was confounded by the significantly younger age of their patients with continent diversion. Younger patients introduce a significant source of bias in favour of continent diversion in that numerous conventional measures of baseline QOL are better in younger patients with less comorbidity. Therefore, equivocal results, or even small improvements in favour of continent diversion in such patients, may be misleading.

The time-tested ileal conduit is deliberately constructed to be incontinent and to minimize the dwell time of urine in bowel. ${ }^{5}$ These were the reasons Bricker developed it as an alternative to the ureterosigmoidostomy. The new alternatives to the conduit, the orthotopic neobladder or the less commonly performed cutaneous "continent" diversion, revert to increasing urine dwell time but not always with the anticipated continence. There are documented significant rates of urinary incontinence after so called continent diversions. Cutaneous diversions suffer failure of the continence mechanism in at least $10 \%$ of cases with intussuscepted nipple valves and up to $28 \%$ of buttressed native ileocecal valves. ${ }^{6,7}$ These rates are based on the total number of procedures done and do not allow for the acturial loss of patients owing to bladder cancer or other disease. Orthotopical diversions lack a normal guarding reflex to increase external sphincter tone as the bladder fills, which can lead to incontinence. Daytime incontinence rates can reach $20 \%$, and nocturnal incontinence rates can be significantly higher. ${ }^{8} \mathrm{~A}$ Danish series ${ }^{9}$ of 166 patients followed after Kock pouch neobladder reported that $55 \%$ were still using a pad or condom device as a nighttime protective device after 3 years. A large series from Studer's group ${ }^{10}$ reported rates of daytime incontinence of $8 \%$ of more than 1300 patients and persistent nighttime incontinence in $20 \%$ at 1 year (virtually all are incontinent initially). It is highly possible that these reported rates significantly underrepresent the reality. Data collection is often conducted by the surgical team, not a third party. The treatment of this incontinence has been problematic as well. The largest available series reported suboptimal results in each of 4 women treated with pubovaginal sling procedures for incontinence after orthotopic neobladder construction. Two were rendered hypercontinent, requiring intermittent catheterization, while 2 suffered sepsis-related deaths subsequent to enterotomy and fistulization following difficult retropubic dissection. ${ }^{11}$

There is very little information about the impact of aging on patients' ability to manage continent urinary diversion. The incidence of bladder cancer increases with age and our population is aging. We are seeing more and healthier elderly patients in whom radical cystectomy can be considered. They may even be candidates for a discussion of continent diversion at the time of surgery. However, those patients who do not die of bladder cancer will inevitably age rapidly, 
which will compromise their ability to manage a neobladder or a cutaneous continent stoma. Increased comorbidity will mean that patients are less capable of coping with any electrolyte or acid-base derangements, drug metabolism alterations or urinary tract infections that they may suffer. Decreased cognitive and physical function would impair effective emptying, catheterization and irrigation of the diversion, which would increase expected complications and place an ever-increasing burden on caregivers. We are unaware of specific reports to confirm or refute this notion, but its logic seems irrefutable.

The large series of continent urinary diversions are published by large tertiary centres with extensive experience with the various techniques of urinary diversion, including their follow-up and outpatient management postoperatively. These results may not be generalizable. Moderate volume or smaller centres may lack the experience or resources required for optimal patient care in continent diversion, despite their ability to achieve excellent and comparable results in cystectomy and conduit creation. Published data on outcomes after continent diversion should thus be interpreted with caution and may not be representative of the probable outcomes in the greater population.

Continent urinary diversion is a more technically challenging and longer operation than conduit creation. This may be associated with an increased complication rate. Longer operating times result in increased blood loss, transfusion requirement and anesthetic complications. Increased length of hospital stay places patients at increased risk of morbidity and increases health care costs. Continent diversions are associated with significant reoperation rates, even into late follow-up periods. The Lund group of Mansson and colleagues ${ }^{12}$ noted $22 \%$ and $23 \%$ reoperation rates for orthotopic and continent cutaneous diversions, respectively, in their series of 166 patients. Series suggesting similar complication or metabolic derangement rates between continent and incontinent diversion are often plagued with the same age bias in favour of continent substitution that are seen in the QOL literature. ${ }^{13}$ The incidence and consequence of these complications in the aging and increasingly frail patients are understudied and probably underappreciated. Reoperation becomes more problematic, and the ability of the aging body to handle chronic metabolic complications is reduced.

As aforementioned, it is important to remember the actuarial impact of loss of patients by death from disease when calculating rates of chronic complications. This will reduce the rates of incontinence and chronic complications. Furthermore, centres performing and publishing results from significant numbers of continent diversion may, in the spirit of inquiry, have a tendency to broaden indications for cystectomy and continent diversion. This might produce a cohort of patients with more advanced or worse prognosis disease. Worse disease will lead to decreased disease-specific survival and create an artificially low rate of complications in subsequent outcome series by increasing the denominator (total patients undergoing diversion) without changing the numerator (absolute number of patients suffering long-term complications). In short, patients will be dead, not incontinent or disabled.

Despite the above, we concede that there are some highly motivated and physically adept women and men who are undergoing cystectomy with the intent to cure good-prognosis disease in an experienced institution with excellent support available both socially and from the health care team at the hospital and in the community. Such men and women would be expected to benefit from a continent diversion. It is these caveats and de facto inclusion criteria, however, that make an excellent case in favour of creating an incontinent diversion in most patients.

It becomes clear when one considers quality of life differences, complication rates, the impact of the aging population, the lack of generalizability of major centre data and the decreased age of continent diversion cohorts, that the benefits of continent diversion may be overstated in the literature and, more importantly, at the time of informed consent. While there is a role for continent diversion in a few well selected patients, it is clear that the incontinent ileal conduit remains the appropriate diversion for most patients undergoing radical cystectomy.

*Fellow, Uro-Oncology Fellowship Program, University of Toronto, Toronto, Ont., †Professor, Division of Urology, Department of Surgical Oncology, Princess Margaret Hospital and the University Health Network, University of Toronto, Toronto, Ont.

The positions provided in the Point/Counterpoint series are presented as general information and do not necessarily reflect the personal opinions of the authors. 
This article has been peer reviewed.

Competing interests: None declared.

\section{References}

1. Porter MP, Wei JU, Penson DF. Quality of life issues in bladder caner patients following cystectomy and urinary diversion. Urol Clin North Am 2005;32:207-16.

2. Hart S, Skinner EC, Meyerowitz BE, et al. Quality of life after radical cystectomy for bladder cancer in patients with an ileal condiut, cutaneous or urethral Kock pouch. J Urol 1999;162:77-81.

3. Hardt J, Filipas D, Hohenfellner R, et al. Quality of life in patients with bladder carcinoma after cystectomy: first results of a prospective study. Qual life Res 2000;9:1-12.

4. Dutta SC, Chang SS, Coffey CS, et al. Health related quality of life assessment after radical cystectomy: comparison of ileal conduit with continent orthotopic neobladder. J Urol 2002;168:164-7.

5. Butcher HR Jr, Sugg WL, McAfee CA, et al. lleal conduit method of urinary diversion. Ann Surg 1962;156:682-91

6. Wiesner C, Stein R, Pahernik S, et al. Long-term follow-up of the intussuscepted ileal nipple and the in situ, submucosally embedded appendix as continence mechanisms of continent urinary diversion with the cutaneous ileocecal pouch (Mainz pouch I).
J Urol 2006; 176:155-9

7. Holmes DG, Thrasher JB, Park GY, et al. Long-term complications related to the modified Indiana pouch. Urology 2002;60:603-6.

8. O'connor RC, Kuznetsov DD, Patel RV, et al. Arrificial urinary sphincter placement in men after cystectomy with orthotopic ileal neobladder: continence, complications, and quality of life. Urology 2002;59:542-5.

9. Steven K, Poulsen AL. The orthotopic Kock ileal neobladder: functional results, urodynamic features, complications and survival in 166 men. J Urol 2000;164:288-95.

10. Hautmann RE, Volkmer BG, Schumacher MC, et al. Long-term results of standard procedures in urology: the ileal neobladder. World I Urol 2006;24:305-14.

11. Quek ML, Ginsberg DA, Wilson S, et al. Pubovaginal sling for stress urinary incontinence following radical cystectomy and orthotopic neobladder reconstruction in women. J Urol 2004;172:219-21.

12. Mansson W, Davidsson T, Konyves J, et al. Continent urinary tract reconstruction the Lund experience. BJU Int 2003;92:271-6.

13. Benson MC, Slawin KM, Wechsler MH, et al. Analysis of continent versus standard urinary diversion. Br J Urol 1992;69:156-62.

Correspondence: Dr. Michael A.S. Jewett, Division of Urology, University of Toronto, 610 University Ave., 3-124, Toronto ON M5G 2C4; m.jewett@utoronto.ca

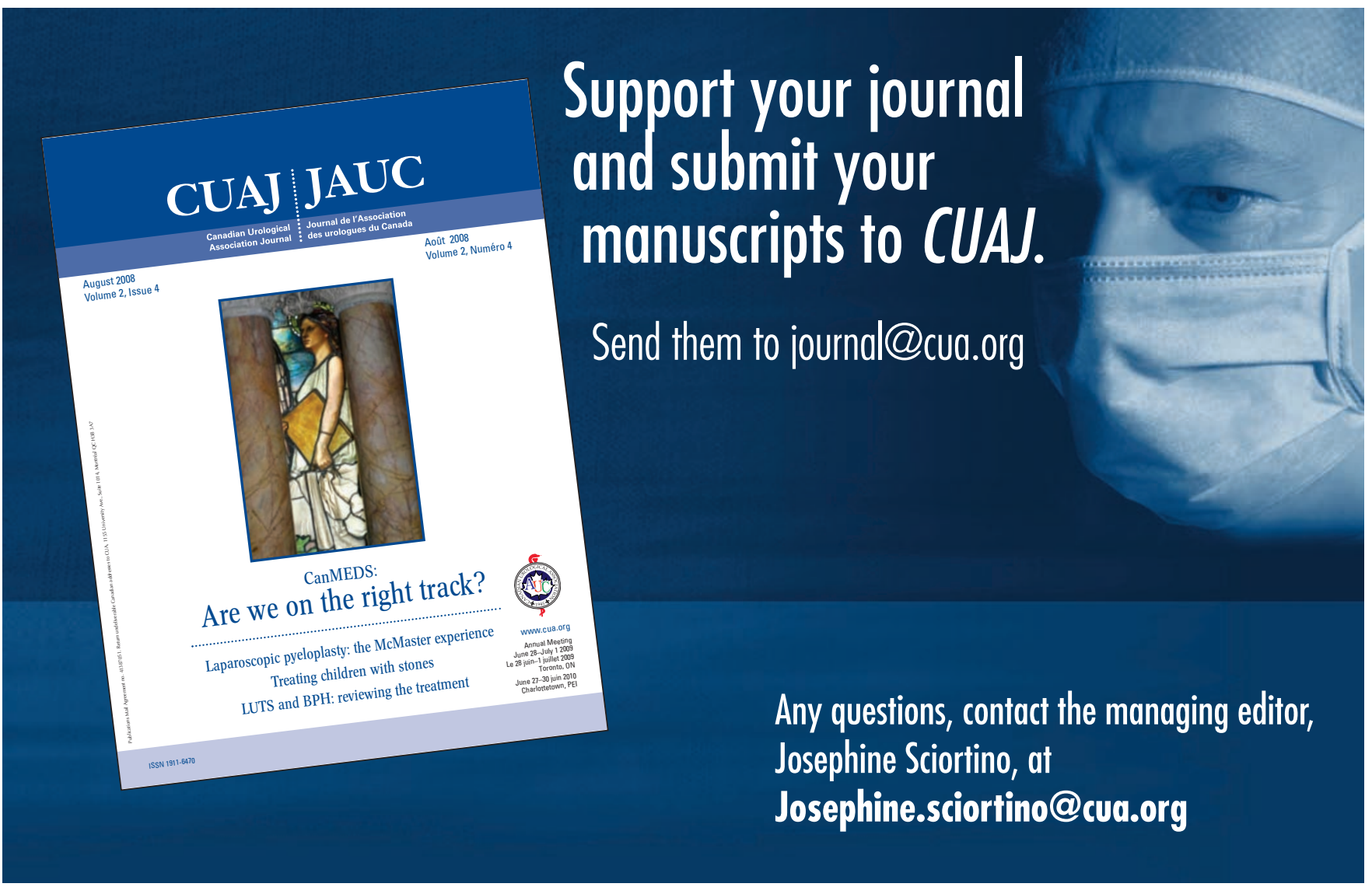

